\title{
Polymorphism of sugars studied by time domain NMR
}

\author{
D. Le Botlan*, F. Casseron and F. Lantier \\ Laboratoire d'Analyse Isotopique et Électrochimique de Métabolismes, \\ UPRESA CNRS Q6006, Faculté des Sciences, BP. 92208, 44322 Nantes Cedex 03, France
}

\begin{abstract}
The $T_{1}$ NMR relaxation time measured by time domain NMR spectroscopy was used to compare the mobility of the solid state of different sugars. To avoid time consuming experiments, a relaxation delay inferior to $5 . T_{1}$ has been used for crystalline samples. In this case, the commonly used relationship can no longer be used. The analysis of relaxation curves by a continuous method (CONTIN program) allowed to obtain quantitative results for crystalline and amorphous phases in mixtures, well related to experimental values. Photos by electronic scanning microscopy of crushed crystalline sucrose show pieces of sucrose with rounded corners, probably due to a partial melting of sucrose during crushing. This layer must be in an amorphous state. However, X-ray diffraction spectra did not show a significant amount of an amorphous phase. The very slight difference in the position of the $T_{1}$ distribution between rough crystalline sucrose and crushed sucrose could be due to the occurrence of an amorphous phase at the surface of the crystals.
\end{abstract}

Key words. Time domain NMR $-T_{1}$ relaxation time - sugars - amorphous phase.

\section{Introduction}

Obtained from either sugar cane or sugar beet, sugar (sucrose) is a food ingredient which is available worldwide. Its use is commonplace both as sugar for the table and as a basic ingredient for the food industry. For domestic con- sumption, it is available as granulated, caster or lump sugar, with the advantage of stable preservation in our climate. For industrial usage, there are numerous forms of sugar including crystalline ("dry" sugar), with a wide range of granulation (icing sugar to candy sugar), and liquid forms (simple solution, invert sugar). 
Under certain conditions of temperature and humidity, insufficiently dried crystalline sugar or finely-crushed sugar can partially set to a mass ("caking"). This phenomenon is attributed in part to a (re)crystallization of sugar present in the amorphous state [1]. The amorphous state is situated outside thermodynamic equilibrium; this metastable state is characterized by an absence of crystallinity, which does not necessarily mean an absence of organization. The crystalline state of sucrose is characterized by a unique structure no matter how it is obtained; this structure was discovered by neutron diffraction [2] and confirmed by X-ray analysis [3]. In contrast, it is generally considered that the molecular organization of amorphous sugar is different for each process which leads to an amorphous structure. Thus, a single analytical technique cannot provide full information [1].

Amorphous sugar can result from dry crushing of crystalline sugar, from rapid cooling or instant drying of sugar solution, or from freeze-drying or extrusion of concentrated solutions followed by cooling. These different methods lead to two types of physical structure, which differ essentially in the degree of humidity and the degree of order of the sample; undercooled liquid (sugar crushed, melted and cooled) or a metastable super-saturated solution said to be an amorphous concentrated solution (sugar atomised, freeze-dried or freeze-concentrated). Extrusion leads to a form intermediate between these two structures. In the case of freeze-drying, Carstensen and Van Scoik [4] have reproduced the organizational state of solid amorphous sugar with drops of sugar solution plunged into liquid nitrogen and freeze-dried. X-ray analysis of freeze-dried sugar detects no crystalline state. In contrast, observation of the same sample by scanning electron microscopy reveals the presence of microcrystals, organized in a lamellar structure. This microcrystallinity is the origin of the instability and the recrystallization of freezedried amorphous sugar when it is subjected to high pressure steam or heat treatment $[5,6]$. In addition to identifying the presence of amorphous sugar, it would be interesting, especially for the sugar industry, to be able to quantify it using a simple analytical technique so that the risk of increased moisture content and "caking" could be predicted.

Different techniques, such as cross polarization magic angle ${ }^{13} \mathrm{C}$ NMR (CP-MAS), differential thermal analysis (DTA), Fourier transform IR spectroscopy (FTIR) and X-ray analysis, can distinguish clearly an amorphous sugar from a crystalline sugar, as well as sugars of different physical structure [5-7]. These methods, however, are not quantitative when applied to a heterogeneous system. Thus, with CPMAS ${ }^{13} \mathrm{C} \mathrm{NMR}$, the most rigid or the most mobile part is favoured according to "time of contact" [8].

Due to its rapidity and ease of use (generally no sample preparation is needed), time domain NMR provides an interesting and effective method for quality control in the food processing industry.

For studying solids, the spin-lattice relaxation time $\left(T_{1}\right)$ has the advantage of being much more sensitive to correlation time $\tau_{\mathrm{c}}$ than the spin-spin relaxation time $\left(T_{2}\right)$. Whether one is dealing with a crystalline or an amorphous form, a solid phase has a $T_{2}$ of the order of $15 \mu \mathrm{s}$ [9]. In contrast, the $T_{1}$ of the $\alpha$ form of tristearin (hexagonal form) has been measured at $250 \mathrm{~ms}$, while that of the $\beta$ form (triclinic form) is $1.7 \mathrm{~s}$ [10]. According to Azouri [10], Norton [11] was the first to use the parameter $T_{1}$ to differentiate polymorphic forms.

Thus, two polymorphic forms can be distinguished using their relaxation times $T_{1}$. This parameter describes the return of the magnetization to equilibrium according to the axis $\mathrm{Oz}$. The $T_{1}$ is only affected by the rapid movements of proton populations near to the Larmor frequency. It gives a minimum value for $\mathrm{w} . \tau_{\mathrm{c}}=1$ [12]; $\tau_{\mathrm{c}}$ corresponds approximately to the average time for a molecule or functional group to turn an angle of one radian. The $T_{1}$ reflects the return to thermal equilibrium between the spin system and the environment.

\section{Materials and methods}

The NMR spectrometer used was a Minispec PCl20, Bruker, with a magnetic field of 0.47 Tesla (proton resonance frequency of $20 \mathrm{MHz}$ ). All the sugar samples were measured under the following experimental conditions: NMR probe VTS $10,2 \mathrm{~cm}$ depth of sample in the tube, measurement temperature of $40{ }^{\circ} \mathrm{C}$ (temperature of the magnet), detection method - "phase sensitive detection" (PSD); attenuation att $=27$; acquisition number, 9; the relaxation delay, RD, was adapted for each measurement according to the results anticipated, 3, 4 and $10 \mathrm{~s}$.

Commercial sugars were used except for glucose and fructose (Sigma) which had to be recrystallized in alcohol (as they may contain paramagnetic or ionic impurities that could perturb the NMR signal). Different forms of sugar were used: crystalline, icing, crushed and/or sifted, "granules" and "instant" sugar. Depending on the process of production, these forms contain varying proportions of sugar in the amorphous state. It should be noted that icing sugar is obtained by fine crushing of crystalline sugar then addition of an anticaking agent, such as silica or a starch derivative. To produce "granules" sugar, moistened crystalline sugar is agglomerated in presses; these agglomerates are then dried and ground coarsely. "instant" sugars are made from icing sugar, agglomerated using steam in an adapted spray tower then dried.

For amorphous sugar, commercial "cooked sugar" obtained by rapid cooling of a super-saturated solution was used. X-ray spectroscopy confirmed that this sugar contains no crystalline phase (Fig. 1). The X-ray spectra were measured on a SIEMENS D5000 spectrometer, working in thetatheta geometry, with a step of $0.03^{\circ}$ and a counting time of $15 \mathrm{~s}$ per degree.

Scanning electron micrographs were obtained using a JEOL JSM $6400 \mathrm{~S}$ field effect microscope.

The relaxation time $T_{1}$ can be measured by two different pulse sequences, called inversion-recovery and progressive saturation. The more commonly-used inversion-recovery sequence was used.

\section{Inversion-recovery method}

The inversion-recovery method consists of inverting the magnetization according to the axis $O z$, then allowing it to evolve for different times $\tau_{i}$. The measurement made according to the axis $O y, 11 \mu \mathrm{s}$ after a pulse at $90^{\circ}$ is given by the sequence: 


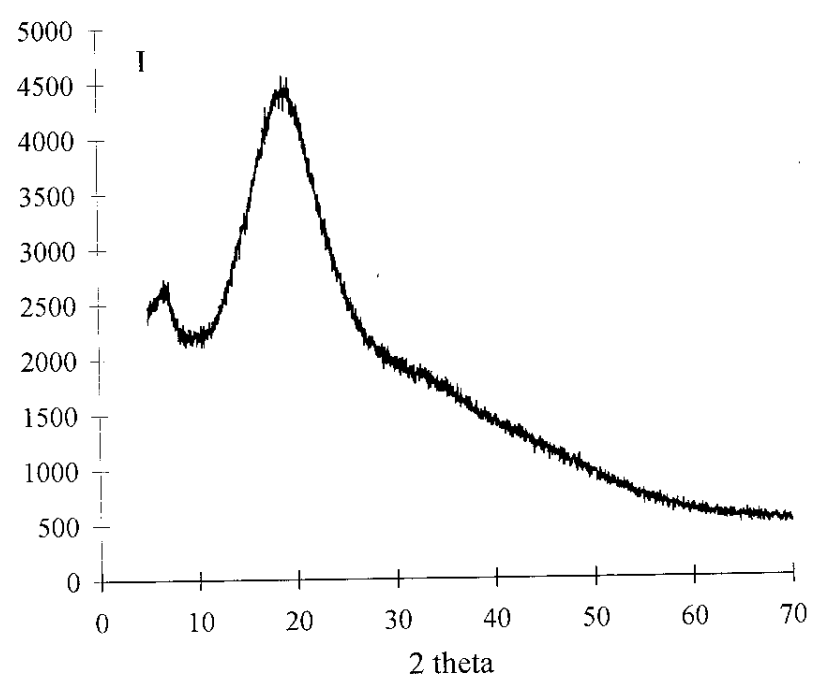

Fig. 1. X-ray diffractogram of amorphous sucrose.

$$
\left(180^{\circ}-\tau_{i}-90^{\circ}-11 \mu \mathrm{s}-\text { measured } i, \mathrm{RD}\right) n
$$

The relaxation curve obtained for amorphous sugar is presented in figure 2 . The magnetization follows the general relation $[13,14]$ :

$$
I=I_{0} \sin \theta_{2} \frac{1-\left[1-\cos \theta_{1}\left(1-\exp \left(-\frac{R D}{T_{1}}\right)\right)\right] \exp \left(-\frac{\tau}{T}\right)}{1-\left[\cos \theta_{1} \cos \theta_{2} \exp \left(-\frac{R D}{T_{1}}\right)\right] \exp \left(-\frac{\tau}{T}\right)}
$$

Under normal (theoretical) conditions, $\theta_{1}=180^{\circ}, \theta_{2}=90^{\circ}$ and the relaxation delay RD is at least 5 times the maximum value of $T_{1}$. Thus, the return to equilibrium of the longitudinal component of the magnetization can be expressed by the simplified relation, commonly used [15]:

$$
I=I_{0}\left[1-2 \exp \left(-\frac{\tau}{T}\right)\right]
$$

However, whatever the efficiency of the pulses, the real angle of tilting of the magnetization vectors is never $180^{\circ}$ or $90^{\circ}$. In fact, the relation should be

$$
I=I_{0}\left[1-a \exp \left(-\frac{\tau}{T}\right)\right]
$$

with $a=1-\cos \left(\theta_{1}\right)$.

The relaxation curves are then analysed by the program CONTIN $[16,17]$. This program has the advantage of determining the distribution of the relaxation times $T_{1}$ (Fig. 3) instead of discrete values as many other programs do.

\section{Results and discussion}

For crystalline solids, the $T_{1}$ is several seconds. As a result, the relaxation delay RD $\left(5 . T_{1}\right)$ becomes very large and the measurement times become much too long for a routine operation.

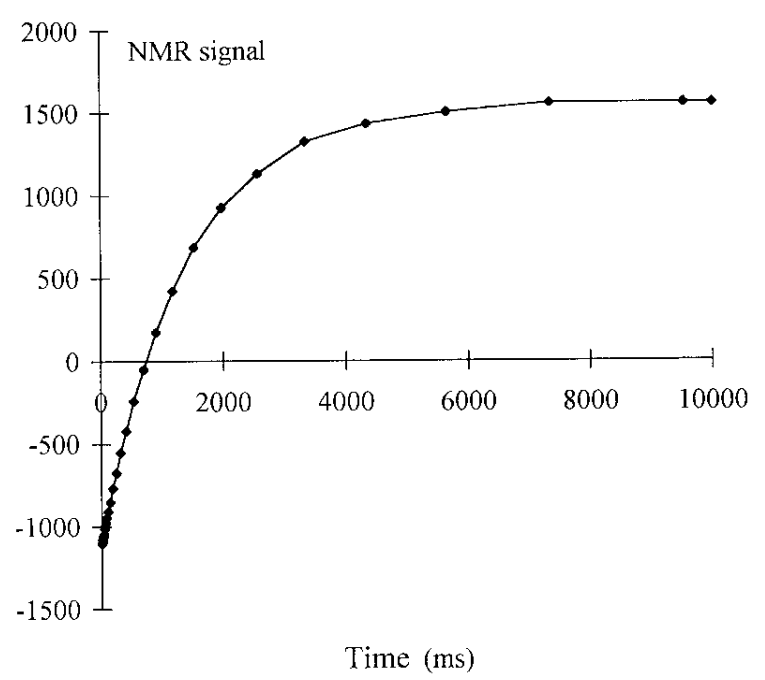

Fig. 2. NMR signal obtained during an inversion-recovery sequence.

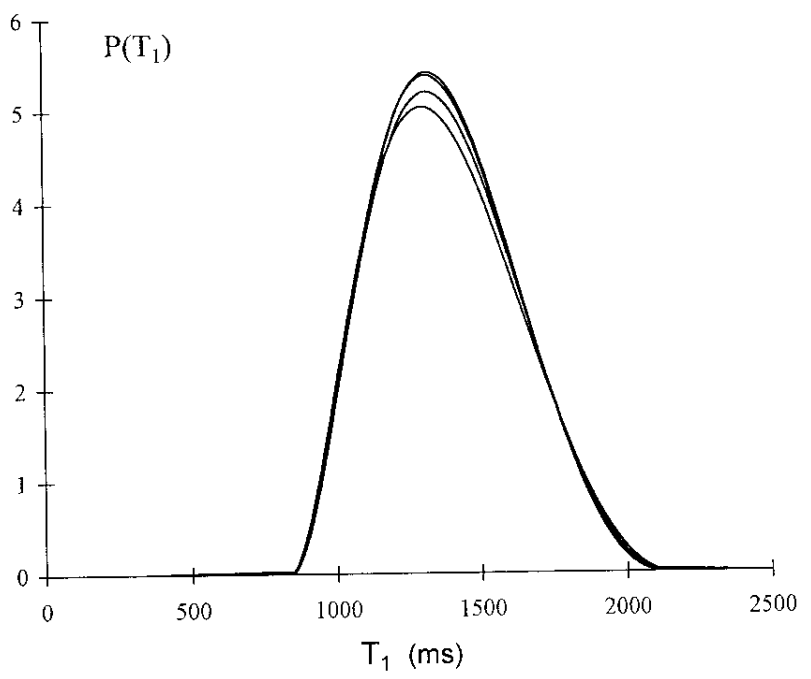

Fig. 3:. Distribution of relaxation times $T_{1}$ obtained with crushed crystalline sucrose.

Experimentally, $\theta_{1}$ and $\theta_{2}$ are never equal to $180^{\circ}$ and $90^{\circ}$. We have previously obtained $\theta_{1}=175^{\circ}$ with a Bruker WM250 [18]. Furthermore, when the relaxation delay RD is less than 5 times $T_{1}$, the term $\exp \left(-\mathrm{RD} / T_{1}\right)$ is no longer negligible and the denominator includes a term $\exp \left(-\tau / T_{1}\right)$ with a coefficient that is no longer null. Simulations with $\theta_{1}=2$. $\theta_{2}=180^{\circ}, 160^{\circ}, 140^{\circ}$ and $\mathrm{RD}=2 T_{1}$ have shown, however, that simulated values can be modeled correctly by the relation $I=I_{0}\left[1-a^{\prime} \exp \left(-\frac{\tau_{i}}{T_{1}}\right)\right]$. Evidently, in this case, $a^{\prime}$ is no longer just the reflection of the imperfection of the pulses. On the other hand, the calculated value of $T_{1}$ is correct to almost $1 \%$. We have verified this experimentally on saccharose with RD $=10 \mathrm{~s}\left(7 T_{1}\right), 4 \mathrm{~s}\left(2.9 T_{1}\right)$ and $3 \mathrm{~s}$ (2.2 $\left.T_{1}\right)$, (Tab. I). 
In the presence of a distribution, the relation is

$$
\begin{gathered}
I=-I_{0} a \int_{0}^{\infty} P\left(T_{1}\right) \exp \left(\frac{-\tau}{T_{1}}\right) \mathrm{d} T_{1}+c s t \\
\text { with } \int_{0}^{\infty} P\left(T_{1}\right) \mathrm{d} T_{1}=1
\end{gathered}
$$

$P\left(T_{1}\right)$ represents the distribution of relaxation times; it may be Gaussian or more likely log-normal.

The constant represents $I_{0}$ and the product $a . I_{0}$ is obtained from the area of the distribution. The ratio of the area to the constant thus gives the factor " $a$ ". The horizontal asymptote of the relaxation curve is arrived at with large values of $\tau_{i}$, more than 5 times $T_{1}$. At the moment of the $90^{\circ}$ pulse, the system is totally relaxed. The value of $I_{0}$ is therefore completely independent of the relaxation delay used.

\section{Repeatability}

In table II, the results obtained for "cooked" sugar with a relaxation delay of $3 \mathrm{~s}$ (about 10 times the relaxation time $T_{1}$ ) are shown. As each preparation technique for amorphous sugar leads to a different kind of organization [1], the value of $T_{1}$ obtained depends on the method of preparation and its level of humidity. For example, we obtained a value of $269.8 \mathrm{~ms}(n=8)$ then $303.9 \mathrm{~ms}(n=5)$ after vacuum drying and water loss of $1.8 \%$ of the total mass. Under rapid conditions for measuring a $T_{1}$, that is with a relaxation delay less than 5 times $T_{1}$, the repeatability of the measurements is equally satisfactory (Tab. III). The distribution of relaxation times $T_{1}$, determined at intervals of several days, is also satisfactory (Fig. 3).

Considering the good repeatability of the signal obtained and the great difference in value between the $T_{1}$ of a crystalline and an amorphous sugar, we hoped to use this method to quantify different solid phases. First, we tested the method on commercial icing sugar containing about 3\% starch (Tab. IV). Although starch has both a crystalline and an amorphous phase in its native state, the crystallites are small enough $(\sim 9 \mathrm{~nm})$ to relax via an amorphous phase. Thus a single distribution for $T_{1}$ is observed for native starch [19] with a maximum at about $150 \mathrm{~ms}$. The values for the areas of distribution obtained by the CONTIN program were corrected to take account of the different proton densities of sucrose and starch and a different relaxation time $T_{2}$ for the solid phase. Despite the small proportion of starch in the mixture, this phase is readily detected by this technique and by the analytical program, with a very satisfactory repeatability of 0.4 points. The X-ray spectrum of a mixture containing $6 \%$ amorphous phase does not reveal this phase at first sight (Fig. 4). The method of Rietveld, however, [20] can detect a small quantity of an amorphous phase.

The presence of an amorphous phase is a problem in crushed crystalline sugar (icing sugar). Scanning electron micrographs show particles with rounded edges, as if the surface had melted. In contrast, untreated crystalline sugar has flat surfaces and sharp angles (Photos 1 and 2). A wide band is not seen in X-ray spectroscopy which would indicate the presence of a significant quantity of an amorphous phase.
Table I. Parameters of relaxation $T_{1}$ obtained with a relaxation delay (RD) of 3, 4 and $10 \mathrm{~s}$, on two samples of crystalline (C) and amorphous (A) sucrose.

\begin{tabular}{ccccc}
\hline Sucrose & $R D$ & $a$ & T1 ms & I0 ua \\
\hline $\mathrm{A} 1$ & 3 & 1.825 & 278 & 1891 \\
$\mathrm{C} 1$ & 3 & 1.724 & 1383 & 1703 \\
$\mathrm{C} 1$ & 10 & 1.813 & 1383 & 1707 \\
$\mathrm{C} 2$ & 4 & 1.770 & 1365 & 1820 \\
$\mathrm{C} 2$ & 10 & 1.825 & 1340 & 1833 \\
\hline
\end{tabular}

Table II. Study of the repeatability of the measurement of $T_{1}$ in "quantitative" conditions, that is with a relaxation delay more than five times $T_{1}$, carried out on amorphous sugar. ${ }^{a}$ ) the measurement step is $17 \mathrm{~ms}$.

\begin{tabular}{ccccc}
\hline$N^{\circ}$ & Surface & $I_{0}$ & $a$ & $T_{1}(m s)^{a}$ \\
\hline 1 & 3354 & 1846 & 1.83 & 293 \\
2 & 3342 & 1841 & 1.82 & 293 \\
3 & 3362 & 1847 & 1.82 & 310 \\
4 & 3357 & 1847 & 1.82 & 310 \\
5 & 3365 & 1847 & 1.82 & 310 \\
\hline
\end{tabular}

Table III. Study of the repeatability of the parameters of relaxation $T_{1},(\mathrm{RD}=3 \mathrm{~s})$ on a sample of commercial sucrose. $\left.{ }^{a}\right)$ as the calculation step is $33 \mathrm{~ms}$, the calculation of the standard deviation has no meaning; this explains why absolutely identical results may be obtained. CV, coefficient of variation.

\begin{tabular}{ccccc}
\hline$N^{\circ}$ & Surface & $I_{0}$ & $a$ & $T_{1}(\mathrm{~ms})^{a}$ \\
\hline 1 & 2953 & 1718 & 1.719 & 1383 \\
2 & 2962 & 1722 & 1.720 & 1383 \\
3 & 2971 & 1723 & 1.724 & 1416 \\
4 & 2974 & 1721 & 1.728 & 1416 \\
5 & 2953 & 1708 & 1.729 & 1383 \\
& & & & \\
Average: & 2963 & 1718 & 1.724 & 1396 \\
$\sigma$ & 9.6 & 6.1 & 0.005 & - \\
$\mathrm{CV}(\%)$ & 0.32 & 0.35 & - & - \\
\hline
\end{tabular}

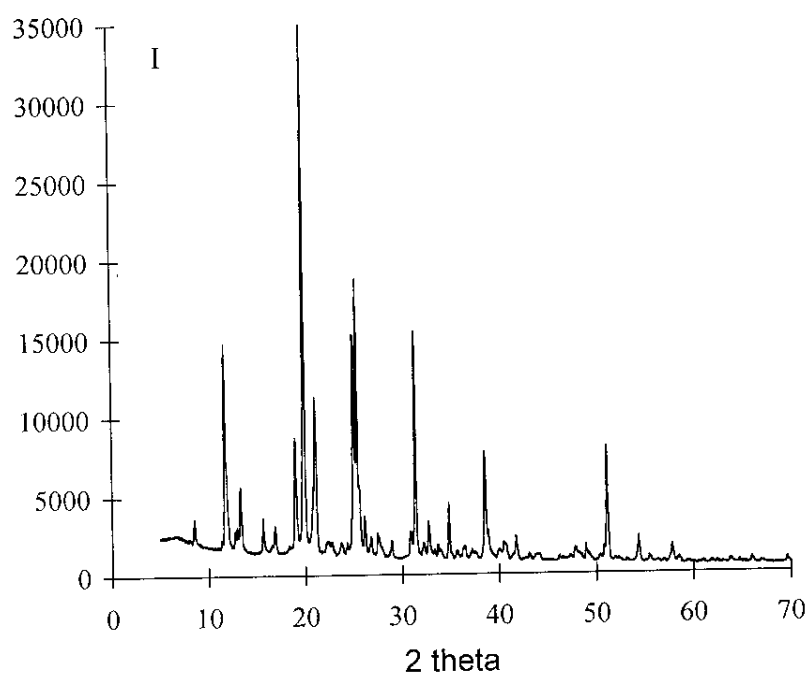

Fig. 4. X-ray diffractogram of a mixture of $6 \%$ amorphous sucrose and $94 \%$ crystalline sucrose. 


\section{Original articles}

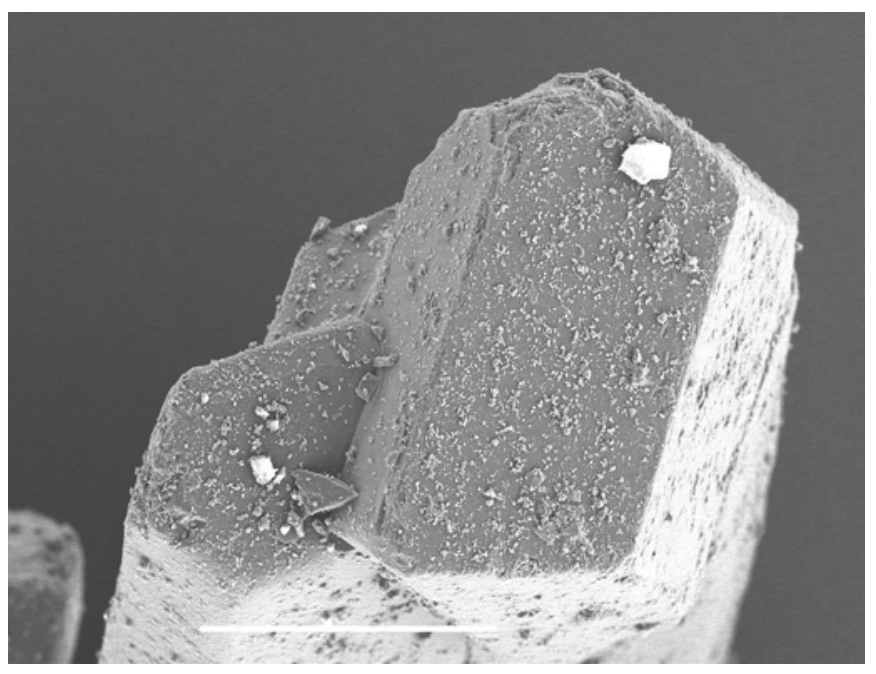

Photo 1. Field-effect scanning electron micrograph of a crystal of sucrose $(-100 \mu \mathrm{m})$.

Table IV. Study of the repeatability of the quantification of the amorphous and crystalline phases, carried out on a sample of icing sugar containing $3 \%$ starch. ${ }^{a}$ ) measurement at $20{ }^{\circ} \mathrm{C}$.

\begin{tabular}{ccccc}
\hline $\mathrm{N}^{\circ}$ & $\begin{array}{c}\text { Amorphous } \\
\text { Fraction \% }\end{array}$ & $\begin{array}{c}\text { Crystalline } \\
\text { Fraction } \%\end{array}$ & $T_{1}(\mathrm{~ms})$ & $T_{l b}(\mathrm{~ms})^{a}$ \\
\hline $1^{\mathrm{a}}$ & 2.80 & 97.20 & 114 & 2087 \\
2 & 2.73 & 97.27 & 105 & 1316 \\
3 & 3.26 & 96.74 & 139 & 1353 \\
4 & 2.73 & 97.27 & 105 & 1316 \\
5 & 3.52 & 96.48 & 139 & 1353 \\
6 & 3.48 & 96.52 & 160 & 1353 \\
Average: & 3.09 & 96.9 & & \\
$\sigma$ & 0.38 & 0.38 & & \\
\hline
\end{tabular}

As different sugars must have different densities in their crystalline state (depending on whether they are mono- or oligosaccharides and on the degree of symmetry of their molecules) we measured the $T_{1}$ of different commercial sugars. Table $\mathrm{V}$ shows that the values of $T_{1}$ range from 1 to $16 \mathrm{~s}$ for crystalline products, other than maltotriose.

Azoury [10] obtained a correlation coefficient of -0.991 between the average $T_{1}$ of a mixture of tristearin and the percentage of the a form in a mixture of $\alpha$ and $\beta$ forms. We wanted to verify that the quantitative aspect of distributions could be applied to mixtures over the whole range of percentages. To do this, we prepared mixtures of crystalline and amorphous sugars. However, due to the different behaviour of these two sugars, it is difficult to obtain a homogeneous mixture. Even after making the mixture in the NMR tube, a

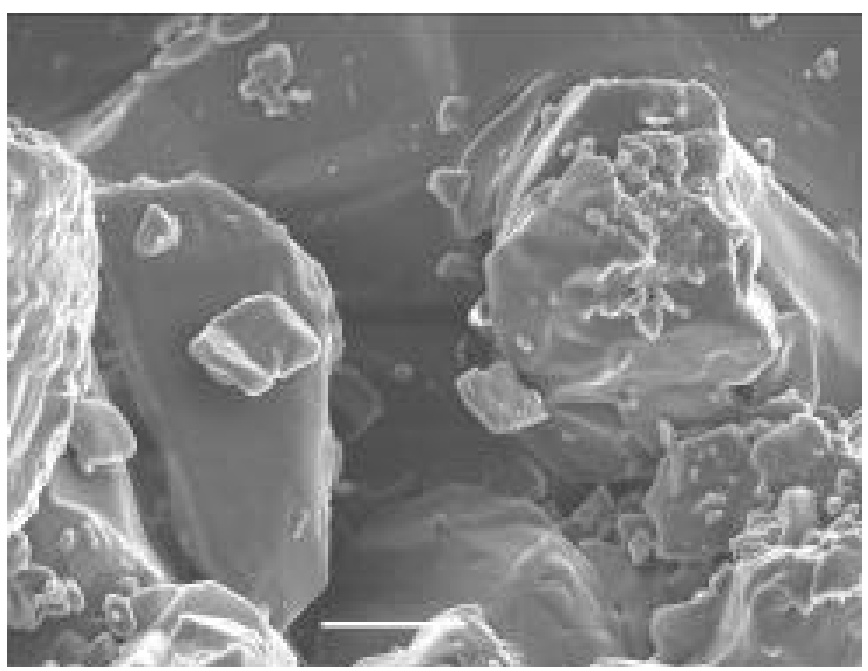

Photo 2. Field-effect scanning electron micrograph of crystals of crushed sucrose $(-100 \mu \mathrm{m})$.

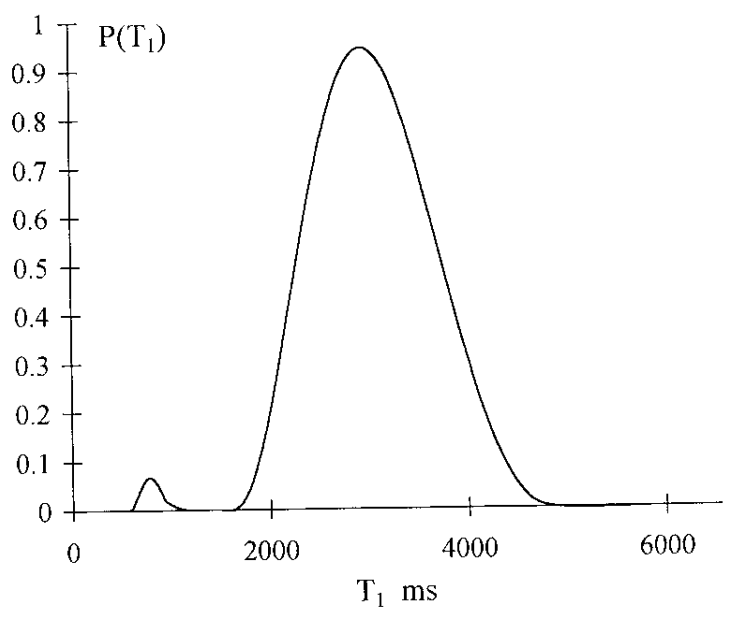

Fig. 5. Distribution of relaxation times $T_{1}$ of a mixture of $6 \%$ amorphous sucrose and $94 \%$ crystalline sucrose.

statistical distribution of the two parts must be done because the NMR signal is not constant throughout both the $2 \mathrm{~cm}$ depth and the thickness of the sample.

In order to limit the measurement time, the relaxation delay was set at $3 \mathrm{~s}$. sufficient to be within the quantitative conditions for measuring amorphous sugar $\left(10 T_{1}\right)$ but very definitely insufficient for the crystalline phase $\left(2.3 T_{1}\right)$. The signal of this phase is minimised to around $10 \%$. The quantities of amorphous and crystalline sugars were obtained from the area in arbitrary units per gram of each of the two pure forms. For four mixtures of between 16 and 70\%, we

Table V. $T_{1}$ of different commercial sugars at $40{ }^{\circ} \mathrm{C}$.

\begin{tabular}{lccccccccc}
\hline sugar & glucose & fructose & lactose & maltose & $\begin{array}{c}\text { Crystalline } \\
\text { sucrose }\end{array}$ & $\begin{array}{c}\text { Icing } \\
\text { sucrose }\end{array}$ & $\begin{array}{c}\text { Instant } \\
\text { sucrose }\end{array}$ & $\begin{array}{c}\text { Amorphous } \\
\text { sucrose }\end{array}$ & $\begin{array}{c}\text { maltotriose } \\
T_{1}(\mathrm{~ms})\end{array}$ \\
\hline 16000 & 4204 & 3714 & 3162 & 1349 & 1383 & 1393 & 295 & 124 \\
\hline
\end{tabular}




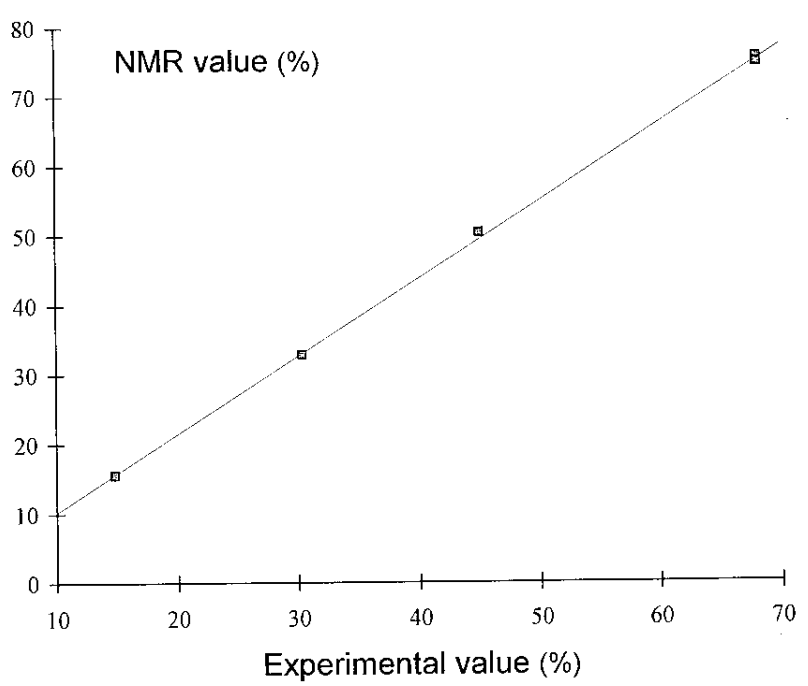

Fig. 6. Change in the percentage of amorphous sucrose determined by NMR in relation to the experimental percentage determined by weighing, in a mixture with crushed crystalline sucrose.

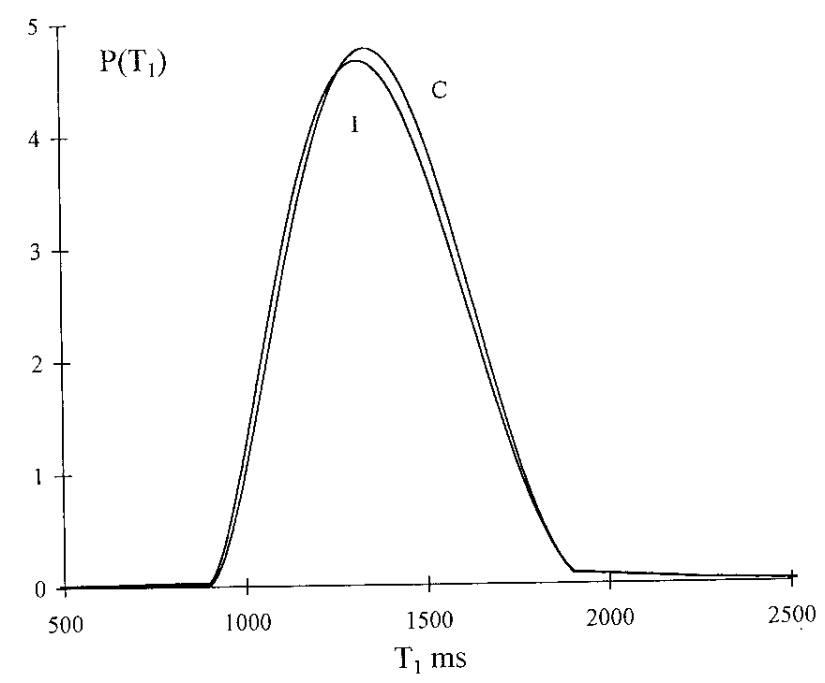

Fig. 8. Comparison of the distribution of relaxation times $T_{1}$ obtained with "instant" (I) and crystalline (C) sucrose.

did not obtain satisfactory absolute values for the quantities of the two phases (Fig. 5 and Tab. VI), but the correlation coefficient between the measured and the experimental percentages is very satisfactory $(\% \mathrm{NMR}=-0.95+1.12 \%$ exp, $r^{2}=0.9995, \sigma=0.67$ ) (Fig. 6).

With "granules" sugar, where a proportion of sucrose has been made soluble then dehydrated, the major distribution corresponds to crystalline sucrose and there is a second distribution at a $T_{1}$ of around $700 \mathrm{~ms}$ (Fig. 7).

We have seen (Fig. 3) that measurements carried out on the same tube give superimposed distributions of relaxation times $T_{1}$, especially with respect to their width. In contrast, the distributions obtained under the same conditions for crystalline sugar and "instant" sugar show a shift both at the level of the maxima and at the edges of the distributions (Fig. 8). Each of these two distributions is the average of

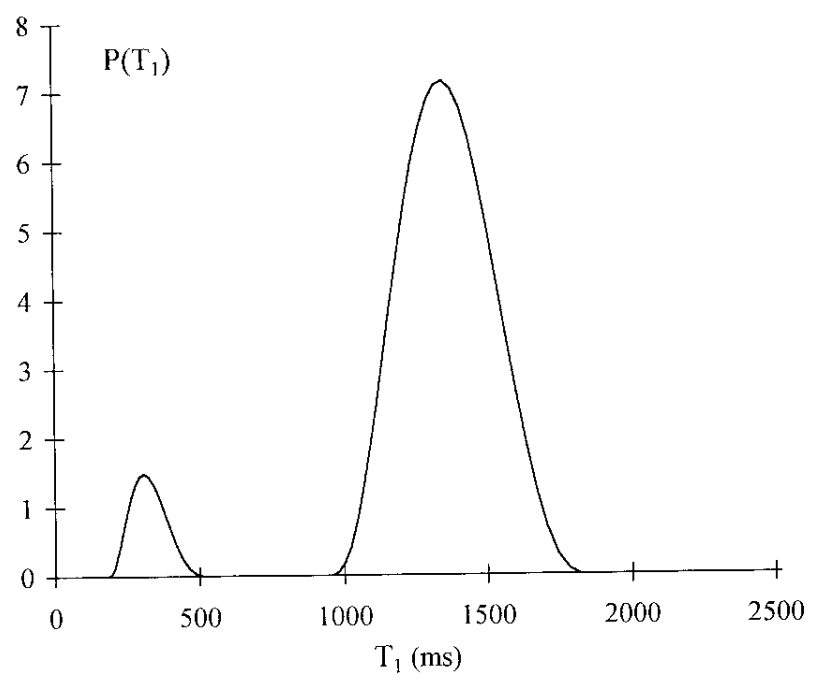

Fig. 7. Distribution of relaxation time $T_{1}$ of "granules" sugar at $20{ }^{\circ} \mathrm{C}$.

Table VI. Comparison of the experimental values and those measured by NMR for the amount of amorphous sucrose in mixtures with crystalline sucrose.

\begin{tabular}{cccc}
\hline \% exp. & $\%$ NMR & Exp.weight $g$ & NMR weight $g$ \\
\hline 14.85 & 15.5 & 0.1742 & 0.17 \\
30.38 & 32.9 & 0.3267 & 0.34 \\
44.97 & 50.4 & 0.4953 & 0.53 \\
68.00 & 75.6 & 0.7448 & 0.88 \\
68.00 & 74.7 & 0.7448 & 0.88 \\
\hline
\end{tabular}

two successive measurements. We calculated the maxima by a $3^{\text {rd }}$ degree polynomial smoothing [21] of the experimental values between 1200 and $1500 \mathrm{~ms}$ and we obtained a value of $1342.1 \mathrm{~ms}$ for crystalline sugar and $1329.8 \mathrm{~ms}$ for "instant" sugar. Thus we consider that "instant" sugar could consist of crystalline sugar covered by a thin layer of amorphous sugar, as seen in photographs of crushed sugar. The hydrogen atoms in the amorphous state, which have greater mobility, could reduce the average relaxation time of those in the crystalline state without constituting a separate population. Considering the typical difference obtained over two consecutive days with crushed sugar (Fig. 3), the difference of $12.3 \mathrm{~ms}$ is significant with a risk of about $1 \%$. (As the characteristics of a spectrometer can change with time and give fluctuating values of $T_{1}$, only the values obtained during a short period must be compared). In this case, the relaxation time observed is given by the relation

$$
\frac{1}{T_{1 \mathrm{~m}}}=\frac{a}{T_{1 \mathrm{c}}}+\frac{1-a}{T_{1 \mathrm{a}}}
$$

$T_{1 \mathrm{c}}$ and $T_{1 \mathrm{a}}$ being the relaxation times of the crystalline and the amorphous forms respectively and $T_{1 \mathrm{~m}}$ the measured relaxation time; " $a$ " represents the proportion of crystalline sugar in the product. With $T_{1 \mathrm{c}}=1342.1 \mathrm{~ms}, T_{1 \mathrm{~m}}=$ $1329.8 \mathrm{~ms}$ and $T_{1 \mathrm{a}}=295 \mathrm{~ms}$, the proportion of crystalline 
sugar obtained is $99.7 \%$. Obviously, this result needs to be confirmed by systematic measurements.

\section{Conclusion}

The spin-lattice relaxation time $T_{1}$, obtained by time domain NMR can be legitimately used to characterize solid phases, particularly for the study of recrystallization in products rich in sugar. By using a relaxation delay less than five times the largest $T_{1}$, quantitative information can be obtained without a prohibitive measurement time.

\section{Acknowledgments}

The authors acknowledge P. Reisser for helpful discussions, A. Barreau for scanning electron micrographs, P. Chevalier for RX spectra, P. Deniard for their interpretation, CEDUS (30, rue de Lubeck, Paris) for financial support and V. Foucault.

\section{Reference}

1. Mathlouthi, M. In: Le saccharose - Propriétés et applications; Mathlouthi, M.; Reiser, P. Ed., Polytechnica, Paris, 1995, Chapter 4.

2. Brown, G. M.; Lévy, H. A. Acta Crystallogr. 1973, B29, 790797.

3. Hanson, J. C.; Sieker, L. C.; Jensen, L. H. Acta Crystallogr. 1973, B29, 797-808.
4. Carstensen, J. T.; Van Scoik, K. G. Pharm. Res. 1990, 7, 12781281.

5. Mathlouthi, M. Ph. D. Thesis, University of Dijon, 1973.

6. Mathlouthi, M. In: Le sucre amorphe : structure, obtention et importance dans les produits alimentaires, III ${ }^{\text {ème }}$ Colloque, Paris, 1994, p 21-31.

7. Mathlouthi, M.; Cholli, A. L.; Koenig, J. L. Carbohydr. Res. 1986, 147, 1-9.

8. Belton, P.; Colquhoun, I. J. Spectr. Int. 1989, 2, 26-33.

9. Tanaka, H.; Takagi, K. British Polym. J. 1989, 21, 519-522.

10. Azoury, R.; Aronhime, J. S.; Sarig, S.; Abrashkin, S.; Mayer, I.; Garti, N. J. Am. Oil Chem. Soc. 1988, 65, 964-967.

11. Norton, I. T.; Lee-Tuffnell, C. D.; Ablett, S.; Bociek, S. M. J. Am. Oil Chem. Soc. 1985, 62, 1237-1244.

12. Abragam, A. In: The principles of Nuclear Magnetism; Oxford University Press Ed., 1961, Chap IV.

13. Freeman, R.; Hill, H. D. J. Chem. Phys. 1969, 51, 3140-3141.

14. Pitner, T. P.; Whidby, J. F. Anal. Chem. 1979, 51, 2203-2206.

15. Martin, M. L.; Martin, G. J.; Delpuech, J. J. In: Practical NMR spectroscopy, Heyden and Son Inc. Ed., Philadelphiia, PA, 1979.

16. Provencher, S. W. Comput. Phys. Commun. 1982, 27, 213-227 and 229-242.

17. Provencher, S. W. J. Chem. Phys. 1976, 64, 2772-2777.

18. Le Botlan, D. J. Chim. Phys. 1987, 84, 115-123.

19. Rugraff, Y., Ph. D. Thesis, University of Nantes, Nantes, Nov. 1996.

20. Rietveld, M. H. P. J. Appl. Cryst. 1969, 2, 65-71.

21. Dennis, J. E.; Gay, D. M.; Welch, R. E. ACM Trans. Math. Software 1981, 7, 3-7. 